\title{
Genome-wide analysis identifies critical DNA methylations within NTRKs genes in colorectal cancer
}

\author{
Zijian Chen ${ }^{1,3+}{ }^{0}$, Zenghong Huang ${ }^{2,3 \dagger}$, Yanxin Luo ${ }^{2,3}$, Qi Zou ${ }^{3,4}$, Liangliang Bai ${ }^{3}$, Guannan Tang ${ }^{3}$, Xiaolin Wang ${ }^{3}$, \\ Guangwen $\mathrm{CaO}^{5}$, Meijin Huang ${ }^{2,3}$, Jun Xiang ${ }^{1 *}$ and Huichuan $\mathrm{Yu}^{3^{*}}$
}

\begin{abstract}
Background: Neurotrophic tropomyosin receptor kinases (NTRKS) are a gene family function as oncogene or tumor suppressor gene in distinct cancers. We aimed to investigate the methylation and expression profiles and prognostic value of NTRKs gene in colorectal cancer (CRC).

Methods: An analysis of DNA methylation and expression profiles in CRC patients was performed to explore the critical methylations within NTRKs genes. The methylation marker was validated in a retrospectively collected cohort of 229 CRC patients and tested in other tumor types from TCGA. DNA methylation status was determined by quantitative methylation-specific PCR (QMSP).

Results: The profiles in six CRC cohorts showed that NTRKs gene promoter was more frequently methylated in CRC compared to normal mucosa, which was associated with suppressed gene expression. We identified a specific methylated region within NTRK3 promoter targeted by cg27034819 and cg11525479 that best predicted survival outcome in CRC. NTRK3 promoter methylation showed independently predictive value for survival outcome in the validation cohort ( $P=0.004, \mathrm{HR} 2.688,95 \% \mathrm{Cl}[1.355,5.333])$. Based on this, a nomogram predicting survival outcome was developed with a C-index of 0.705 . Furthermore, the addition of NTRK3 promoter methylation improved the performance of currently-used prognostic model (AIC: 516.49 vs 513.91; LR: 39.06 vs 43.64, $P=0.032$ ). Finally, NTRK3 promoter methylation also predicted survival in other tumors, including pancreatic cancer, glioblastoma and stomach adenocarcinoma.
\end{abstract}

Conclusions: This study highlights the essential value of NTRK3 methylation in prognostic evaluation and the potential to improve current prognostic models in CRC and other tumors.

Keywords: NTRKS, NTRK3, Methylation, Colorectal cancer, Prognosis

*Correspondence: xiangjun@mail.sysu.edu.cn; yuhch5@mail.sysu.edu.cn †Zijian Chen and Zenghong Huang contributed equally to this work ${ }^{1}$ Department of Gastrointestinal Surgery, The Sixth Affiliated Hospital, Sun Yat-sen University, Guangzhou, China

${ }^{3}$ Guangdong Institute of Gastroenterology, Guangdong Provincial Key Laboratory of Colorectal and Pelvic Floor Disease, The Sixth Affiliated Hospital, Sun Yat-sen University, Guangzhou, China

Full list of author information is available at the end of the article

\section{Introduction}

Colorectal cancer (CRC) is the second leading cause of cancer death over the past years [1,2]. Survival outcomes and optimal regimens vary in CRCs. At this point, some clinicopathological risk factors, such as TNM stage, tumor size, and tumor differentiation, have been used to stratify the risk of CRC death. Unfortunately, they fail to accurately distinguish patients with different outcomes [3], and several molecular biomarkers are being investigated and applied in current

c) The Author(s) 2021. This article is licensed under a Creative Commons Attribution 4.0 International License, which permits use, sharing, adaptation, distribution and reproduction in any medium or format, as long as you give appropriate credit to the original author(s) and the source, provide a link to the Creative Commons licence, and indicate if changes were made. The images or other third party material in this article are included in the article's Creative Commons licence, unless indicated otherwise in a credit line to the material. If material is not included in the article's Creative Commons licence and your intended use is not permitted by statutory regulation or exceeds the permitted use, you will need to obtain permission directly from the copyright holder. To view a copy of this licence, visit http://creativecommons.org/licenses/by/4.0/. The Creative Commons Public Domain Dedication waiver (http://creativecommons.org/publicdomain/zero/1.0/) applies to the data made available in this article, unless otherwise stated in a credit line to the data. 
models to increase their prognostic values $[4,5]$. CRC arises with the accumulation of gene mutations and epigenetic alterations [6, 7]. Among them, the aberrant methylation in gene promoters is prevalent across multiple cancers, which can lead to the inactivation of tumor suppressor genes [8]. Some of these aberrant methylations have been discovered and used to serve as prognostic biomarkers for CRC $[9,10]$.

Neurotropic tropomyosin receptor kinase (NTRK) gene family, including NTRK1, NTRK2 and NTRK3, encodes tropomyosin receptor kinases (TRK), which can induce cell proliferation, differentiation, apoptosis, and survival of neurons through the PI3K, RAS/MAPK/ ERK and phospholipase C-gamma signalling transduction pathways $[11,12]$. The aberrations of NTRKs gene function were widely known to play an oncogenic role in multiple cancers. Among them, NTRKs gene fusion was the best-characterized aberration, which promotes tumorigenesis through the constitutive activation of downstream cell growth and proliferative pathways [12]. The first TRK inhibitor, larotrectinib, has been approved by FDA for the treatment of advanced solid tumors with NTRKs gene fusion [13].

Similar to gene fusion, the aberrant expression of NTRKs gene is a critical event in cancers. NTRK1 promoted proliferation and metastasis of cancer cells and lead to poor prognosis in multiple cancers [14-18], while it suppressed cell proliferation in neuroblastoma [19]. NTRK2 was shown to serve as an oncogene in multiple cancers [20-23], and its increased expression was associated with poor outcome [24, 25]. Based on this, inhibition of NTRK2-encoded TRKB was shown to induce antitumor effects and cellular apoptosis [26, 27]. Similar to NTRK1, NTRK3 has been demonstrated to be an oncogene in breast cancer and gastric cancer $[28,29]$, but it acts as a tumor suppressor gene in CRC, neuroblastomas, and head and neck squamous cell carcinoma [11, 30, 31].

The expression of NTRKs gene can be modulated by promoter methylation. A hypermethylated NTRKs gene promoter is associated with suppressed expression in multiple cancers, such as CRC [11, 32], neuroblastoma [33], glioma [34], ovarian cancer [35] and prostate cancer [36]. Given the critical role of NTRKs gene in multiple cancers, we therefore aimed to perform a comprehensive analysis of NTRKs gene on their methylation signature, expression profile and prognostic value in CRC using the methylation profile we previously established and the published dataset, and identify the optimal CpG sites from NTRKs gene region as methylation biomarkers that can be applied in the current clinical models of CRC to improve their prognostic values.

\section{Materials and methods}

\section{Study cohorts}

In the discovery set, the clinical information and methylation profiles of CRC tissues and matched normal tissues we previously established in Fred Hutchinson Cancer Research Center cohort (FHCRC cohort, GSE48684 [32], $\mathrm{n}=105$, normal $=41$, cancer $=64$ ) using Illumina Infinium HumanMethylation450 BeadChip (450K microarray) were combined with datasets of TCGA-COAD\&READ (The Cancer Genome Atlas-colon and rectum adenocarcinoma, $\mathrm{n}=326$, normal $=41$, cancer $=285$ ) cohort [37] and three Gene Expression Omnibus cohorts (GSE83889 [38], $\mathrm{n}=136$, normal $=35$, cancer $=101$; GSE39582 [39], $\mathrm{n}=585$, normal $=19$, cancer $=566$; GSE87211 [40], $\mathrm{n}=363$, normal $=160$, cancer $=203$ ) to investigate methylation and expression profiles of NTRKs gene, and identify the critical CpG methylations within NTRKs genes in CRC.

For the validation cohorts, we included 229 patients with histologically confirmed, stage I-IV CRC who underwent curative resection at the Sixth Affiliated Hospital of Sun Yat-sen University between 2009 and 2012. The patients were selected according to the exclusion criteria, including hereditary cancer, inflammatory bowel disease, and multiple primary cancers. To avoid a potential effect of chemotherapy on genomic methylation status, patients received chemotherapy before curative resection when tissue sample was collected were excluded. Patients were treated and followed according to the National Comprehensive Cancer Network guideline-based institutional protocol as previously described [41-43.]. Briefly, patients were followed at least every 3 months for the first 2 years and every 6 months for years three to five, in which cancer recurrence was screened by physical examination and cancer biomarkers, and a sequential computerized tomography scan with evidence of the disease followed by biopsy was applied to confirm the recurrence. To externally validate the findings in colorectal cancer and other cancers, we used the methylation array data and clinical information of 23 TCGA cohorts including a colon cancer cohort, a rectal cancer cohort and 21 cohorts of other tumor types, which was referred as external validation set.

The cohort disposition for data analysis was illustrated in Fig. 1. The Institutional Review Board at the Sixth Affiliated Hospital of Sun Yat-sen University approved this study, and all the included patients have been given the written informed consent.

\section{Tissue collection}

Formalin-fixed, paraffin-embedded (FFPE) CRC tissue specimens were available from the pathology registry. 


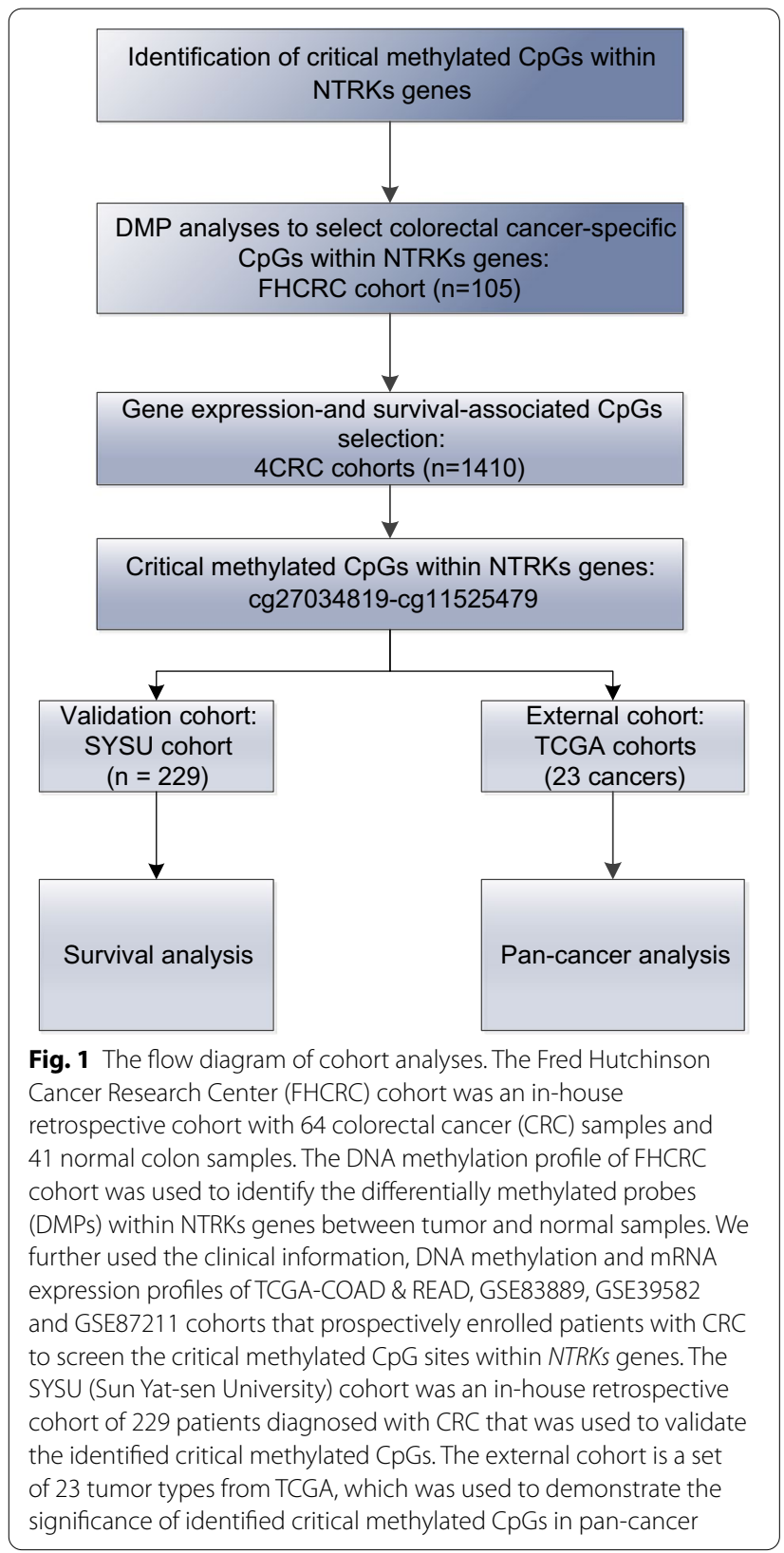

Hematoxylin and eosin (H\&E) stained slides of cancer tissues were assessed by two experienced pathologists to confirm tumor histology based on the WHO classification of cancers and Fuhrman grade. Information of clinicopathological characteristics, such as age at diagnosis, gender, tumor size, tumor differentiation, and TNM stage, were extracted from the pathological reports and medical records.

\section{DNA isolation and methylation analysis}

Genomic DNA from FFPE tissue was isolated using the QIAamp DNA Mini Kit (Qiagen) and bisulfite-treated using EZ DNA Methylation Kit (Zymo) according to the manufacturer's instructions. Gene methylation was analyzed by quantitative methylation-specific PCR (QMSP) that was performed using an ABI Prism 7700 detection system (Biosystems) as previously described [11, 44]. The oligo sequences of primers and probes were presented in Additional file 1: Table S1. The primers and probe designed to target cg27034819 and cg11525479 were illustrated in Fig. 2e. Alu-C4 was used as a reference locus for normalization for input DNA. Each reaction mix was consisted of $0.45 \mu \mathrm{M}$ primers, $0.15 \mu \mathrm{M}$ probe, $1.5 \mathrm{mM} \mathrm{MgCl} 2,200 \mathrm{pM}$ dNTPs, and $1.5 \mathrm{U}$ HotStart Taq. The thermocycler conditions were as follows: $95{ }^{\circ} \mathrm{C}$ for $10 \mathrm{~min}$ followed by $45 \mathrm{cycles}$ of $30 \mathrm{~s}$ at $94^{\circ} \mathrm{C}, 30 \mathrm{~s}$ at $60^{\circ} \mathrm{C}$. All samples were run in duplicate in at least two independent reactions. The methylation status of each sample was determined using the percentage of methylated reference (PMR) method [11]. The $P M R \geq 50$ and $<50$ were defined as hypermethylation and hypomethylation respectively in QMSP assay.

\section{Molecular characterization of CRC}

The CpG Island Methylator Phenotype (CIMP) status and microsatellite instability (MSI) status of a subset of the colorectal neoplasms were assessed as described previously [45]. The assessment of KRAS and BRAF mutation was performed in the Molecular Diagnostic Laboratory

(See figure on next page.)

Fig. 2 The methylation and expression profiles of NTRKs gene in CRC. a-c. The distribution and correlation of methylation and expression profiles of NTRK1, NTRK2 and NTRK3 gene in CRC and normal mucosa tissue. The heatmaps showed the results of probe-dimensional hierarchical clustering analysis of FHCRC cohort based on $\beta$ values of all probes within NTRK1 (a, left), NTRK2 (b, left), and NTRK3 (c, left). Each row represented a probe and each column represented a CRC or normal mucosa sample in the heatmap. Probes targeting promoter region were annotated on heatmap left. The red lines annotated in the heatmap for NTRK3 indicated cg27034819 (top) and cg1 1525479 (bottom). NTRKs gene expression signature in each cohort showed suppressed mRNA expression of NTRK2 (b, middle) and NTRK3 (c, middle) in CRC tissues compared with normal mucosa tissue. Differences between CRC tissue and normal mucosa tissue were assessed with student t-test, and $\mathrm{P}$ values summarized with asterisks were shown in the upper space of the plot $\left.{ }^{* * *} P<0.001\right)$. The Spearman correlation analysis between mean promoter methylation of gene expression in each NTRK gene was shown in right panel. $\mathbf{d}$ Volcano plot of statistical significance against hazard ratio for DFS among all CpG sites within NTRKs gene targeted by $450 \mathrm{~K}$ microarray probes. The top-ranked significantly probe was annotated according to the $P$ values in the univariate Cox analyses. See Additional file 1 for the full results of univariate Cox analysis relevant to this panel. e. A QMSP assay was developed using primers and probe targeting cg27034819-cg11525479 region 

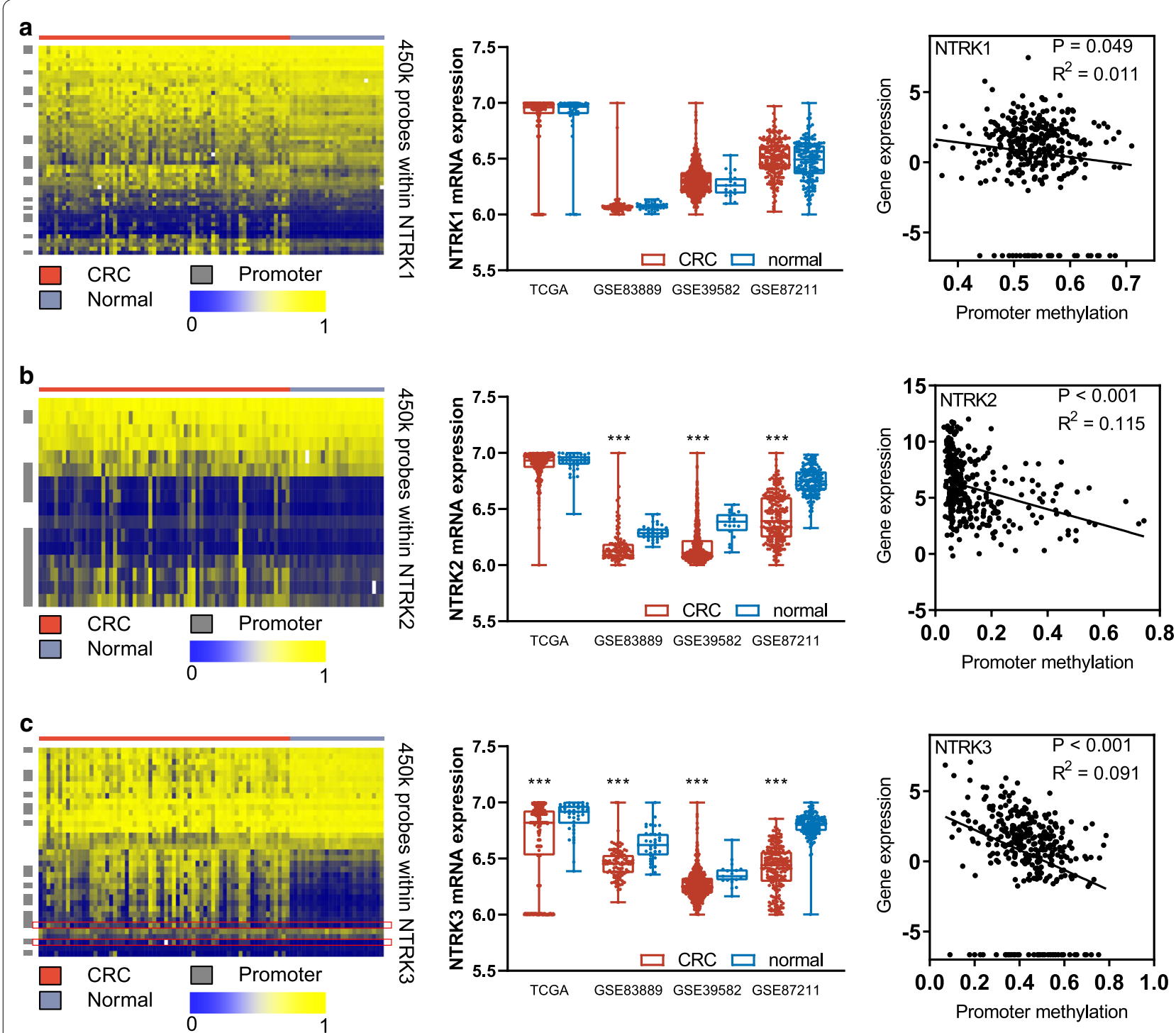

d NTRKs Methylation and CRC Death

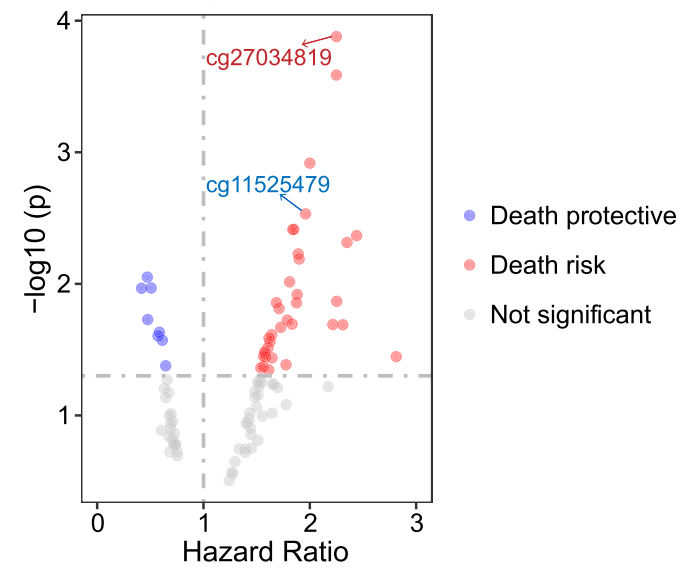

e

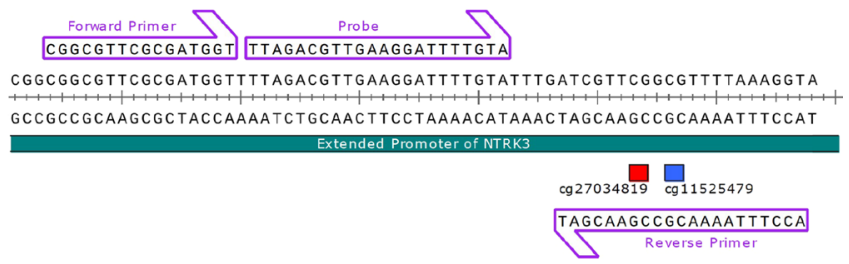


of the Sixth Affiliated Hospital of Sun Yat-sen University, as previously described [46].

\section{Statistical analysis}

All the statistical analyses were conducted using SPSS 20 or R 3.6.1. The NTRKs genes mRNA expression between $\mathrm{CRC}$ tissues and normal tissues were compared using student $\mathrm{t}$-test. The Spearman correlation analysis was used to explore the relationship between promoter methylation and gene expression of NTRKs genes in TCGA cohort. The characteristics of CRC cases with and without NTRK3 promoter hypermethylation were compared using the Wilcoxon rank-sum tests or Chi-square tests. Kaplan-Meier curves and Log-rank tests were used to evaluate the prognostic factors of disease-free survival (DFS) among candidate variables. DFS was defined as the time from curative resection until local recurrence, distant metastasis, or last follow-up. In addition, Cox proportional hazards analyses were used to obtain HRs and corresponding 95\% confidence intervals (CI) for the association between NTRK3 promoter hypermethylation and DFS. The variables that were considered clinically relevant or showed a significant difference $(P<0.05)$ in univariate Cox regression were entered into multivariate Cox proportional-hazards regression model using the backward stepwise selection method. Based on this model, nomograms subjected to internal validation set were generated for predicting 3-year and 5-year DFS outcomes, and the concordance index (C-index) was calculated to evaluate the predictive accuracy. The comparison between NTRK3 hypermethylation and known prognostic factors was assessed using likelihood ratio (LR) and Akaike information criterion (AIC) in competing models including or not including NTRK3 hypermethylation. In general, the model with a lower AIC and a higher LR is considered a better model. To validate these findings in colorectal and other cancers, the univariate Cox regression analysis of NTRK3 hypermethylation on survival outcome was re-performed in 23 TCGA cohorts. In TCGA dataset, the normalized $\beta$ value of cg27034819 and cg11525479 (adjacent to cg27034819), targeting the downstream region of the promoter region in NTRK3, was extracted from $450 \mathrm{~K}$ microarray and its association with survival outcome was analyzed. A P value $<0.05$ was considered statistically significant with a two-tailed test.

\section{Results}

\section{NTRKs gene was commonly suppressed by DNA} methylation in CRC

Using the methylation profile we previously generated through $450 \mathrm{~K}$ microarray, we found NTRKs gene, including NTRK1, NTRK2 and NTRK3, had more frequently methylated promoters in CRC samples when compared with matched normal mucosae (NTRK1, cancer $=0.444$, normal $=0.397, P=0.012$; NTRK2, cancer $=0.251$, normal $=0.167, \quad P<0.001 ; \quad N T R K 3$, cancer $=0.395$, nor$\mathrm{mal}=0.144, P<0.001$; Fig. 2; Additional file 1: Table S2). In support of methylation analysis results, we found the mRNA expression of NTRK2 and NTRK3 in CRC samples was commonly lower than that in normal mucosae using the expression profiles in four CRC cohorts $(n=1410)$. However, no significant difference was observed in NTRK1 mRNA expression (Fig. 2). Moreover, the expression of NTRKs gene is negatively correlated with their mean methylation of promoter (NTRK1, $P=0.049, \quad \mathrm{R}^{2}=0.011 ; \quad$ NTRK $2, \quad P<0.001, \quad \mathrm{R}^{2}=0.115 ;$ NTRK3, $P<0.001, \mathrm{R}^{2}=0.091$; Fig. 2).

\section{A specific methylated region within NTRK3 promoter best predicted CRC death}

We next sought to identify the CPG site that could best predict CRC death and be feasibly used in a clinical assay. In the Cox proportional hazards analyses of $450 \mathrm{~K}$ microarray probes targeting genomic loci within NTRKs gene, we found that the methylation of most CpG sites targeted by these probes was associated with poor survival outcomes in CRC (Fig. 2d, Additional file 1: Table S3). Among them, the methylation of cg27034819 was topranked for predicting CRC death. Of note, we found the probe $\operatorname{cg} 11525479$ was very close to $\operatorname{cg} 27034819$ in their targeting loci (Fig. 2e), and the methylation of cg11525479 also had a predictive value for CRC death that was superior to most probes. These results suggested that the specific region within NTRK3 promoter targeted by cg27034819 and cg11525479 could be used to stratify the death risk of CRC. Interestingly, the methylation of this specific region was shown to be associated with the loss of NT3-dependent tumor suppressor gene function of NTRK3 in our previous in vitro and in vivo study [11]. Therefore, a QMSP assay for determining the methylation of this specific region within NTRK3 promoter was developed (Fig. 2e).

\section{Cohort validation of NTRK3 methylation for prognostic significance}

We further validated the predictive value of this candidate region within NTRK3 promoter in our institutional cohort. The baseline characteristics of this validation cohort were summarized in Table 1 . NTRK3 promoter hypermethylation was observed in 26 of 229 patients $(11.35 \%)$, and it was more frequent in patients with MSI $(P=0.015$; Table 1$)$. Moreover, NTRK3 promoter hypermethylation was associated with KRAS mutation $(P=0.001$; Table 1$)$. For other characteristics relevant to clinical outcomes of CRC, they did not show significant difference between patients with NTRK3 promoter 
Table 1 Baseline characteristics and NTRK3 promotor methylation status among all patients with CRC in validation cohort

\begin{tabular}{|c|c|c|c|c|}
\hline \multirow[t]{2}{*}{ Variables } & \multirow[t]{2}{*}{ Total } & \multicolumn{2}{|c|}{ NTRK3 promotor methylation } & \multirow[t]{2}{*}{$P$ value } \\
\hline & & Hypomethylation & Hypermethylation & \\
\hline \multicolumn{5}{|c|}{ Age (median = 62 years) } \\
\hline$<62$ & 111 & 100 & 11 & \\
\hline$\geq 62$ & 118 & 103 & 15 & 0.504 \\
\hline \multicolumn{5}{|l|}{ Sex } \\
\hline Female & 100 & 88 & 12 & \\
\hline Male & 129 & 115 & 14 & 0.786 \\
\hline \multicolumn{5}{|l|}{ Tumor localization } \\
\hline Colon & 126 & 114 & 12 & \\
\hline Rectum & 103 & 89 & 14 & 0.334 \\
\hline \multicolumn{5}{|c|}{ Tumor size $($ median $=4.5 \mathrm{~cm}$ ) } \\
\hline$<4.5$ & 104 & 90 & 14 & \\
\hline$\geq 4.5$ & 123 & 111 & 12 & 0.382 \\
\hline Unknown & 2 & & & \\
\hline \multicolumn{5}{|l|}{ Tumor differentiation } \\
\hline High or moderate & 191 & 168 & 23 & \\
\hline Poor & 38 & 35 & 3 & 0.584 \\
\hline \multicolumn{5}{|c|}{ Lymphovascular invasion } \\
\hline No & 210 & 188 & 22 & \\
\hline Yes & 17 & 13 & 4 & 0.219 \\
\hline Unknown & 2 & & & \\
\hline \multicolumn{5}{|l|}{ Perineural invasion } \\
\hline No & 208 & 183 & 25 & \\
\hline Yes & 19 & 18 & 1 & 0.611 \\
\hline Unknown & 2 & & & \\
\hline \multicolumn{5}{|l|}{ TNM stage } \\
\hline$|-| \mid$ & 143 & 123 & 20 & \\
\hline III-IV & 85 & 79 & 6 & 0.112 \\
\hline Unknown & 1 & & & \\
\hline \multicolumn{5}{|l|}{ CIMP } \\
\hline Negative & 222 & 198 & 24 & \\
\hline Positive & 7 & 5 & 2 & 0.145 \\
\hline \multicolumn{5}{|l|}{ Microsatellite status } \\
\hline MSS & 125 & 113 & 12 & \\
\hline MSI & 51 & 39 & 12 & 0.015 \\
\hline Unknown & 53 & & & \\
\hline \multicolumn{5}{|l|}{ KRAS } \\
\hline Wild-type & 119 & 110 & 9 & \\
\hline Mutation & 62 & 46 & 16 & 0.001 \\
\hline Unknown & 48 & & & \\
\hline \multicolumn{5}{|l|}{ BRAF } \\
\hline Wild-type & 174 & 151 & 23 & \\
\hline Mutation & 8 & 6 & 2 & 0.674 \\
\hline Unknown & 47 & & & \\
\hline \multicolumn{5}{|l|}{ Ki-67 } \\
\hline$\leq 25 \%$ & 100 & 89 & 11 & \\
\hline$>25 \%$ & 84 & 72 & 12 & 0.502 \\
\hline Unknown & 45 & & & \\
\hline
\end{tabular}


Table 1 (continued)

\begin{tabular}{lclc}
\hline Variables & Total & NTRK3 promotor methylation & \\
\cline { 4 - 4 } & & Hypomethylation & Hypermethylation \\
\hline CA19-9 & 179 & 160 & 19 \\
$\leq 37$ & 32 & 29 & 3 \\
$>37$ & 18 & & \\
Unknown & 158 & 138 & 20 \\
CEA & 57 & 54 & 3 \\
$\leq 5$ & 14 & & \\
Unknown & 15 & & \\
\hline
\end{tabular}

CIMP: CpG island methylator phenotype; KRAS: kirsten rat sarcoma viral oncogene; BRAF: B-Raf proto-oncogene, serine/threonine kinase; Ki-67: kiel67 antigen; CA19-9: carbohydrate antigen 19-9; CEA: carcinoembryonic antigen

hypermethylation and hypomethylation, including age, sex, tumor size, tumor differentiation, lymphovascular invasion, perineural invasion, TNM stage, CIMP, $B R A F$ mutation, Ki-67, CA19-9, and CEA.

In the Kaplan-Meier curve, significantly worse DFS outcomes were observed in patients with NTRK3 promoter hypermethylation compared to those with NTRK3 promoter hypomethylation $(P=0.012$; Fig. $3 \mathrm{a})$. The prognostic value of NTRK3 promoter methylation status was further confirmed by univariate Cox proportional hazards $(P=0.014$, HR $2.194,95 \%$ CI $[1.169,4.117]$; Table 2$)$. Next, in the light of multivariate analysis, NTRK3 promoter hypermethylation was still a prognostic factor adjusted by age, TNM stage, and BRAF mutation $(P=0.004$, HR 2.688, 95\% CI [1.355, 5.333]; Table 2).

In sensitivity analyses, NTRK3 promoter methylation was still independently associated with poor DFS outcome after the exclusion of patients with stage IV disease $(P=0.015$, HR $2.630,95 \%$ CI $[1.206,5.734]$; Fig. 3b, Table 2), CIMP $(P=0.003$, HR $2.806,95 \%$ CI [1.432, 5.500]; Additional file 1: Table S4), MSI $(P=0.008$, HR 3.483, 95\% CI [1.391, 8.717]; Table Additional file 1: S5), or BRAF mutation $(P=0.025, \mathrm{HR} 2.603,95 \% \mathrm{CI}[1.125$, 6.022]; Additional file 1: Table S6) in multivariate Cox analysis.

\section{A nomogram for predicting DFS in CRC patients}

A nomogram for predicting 3-year and 5-year DFS outcome was generated using the variables from the multivariate Cox model, including NTRK3 methylation, age at diagnosis, TNM stage, and BRAF mutation (Fig. 3c). The calibration curves for the nomogram were shown, and the C-index of the nomogram for predicting DFS was 0.705 (Fig. 3d).

\section{NTRK3 methylation adds values to current prognostic panels}

The model 1 had a lower AIC and a higher LR compared with the model 2 (AIC: 597.73 vs 600.69; LR: 6.91 vs 5.95, $P=0.005$; Table 3), indicating that NTRK3 hypermethylation alone is better in predicting prognosis than rough TNM staging alone. In the comparison between model 2 and 3, after NTRK3 hypermethylation was added to TNM stage, a lower AIC and a higher LR were observed

\section{(See figure on next page.)}

Fig. 3 Prognostic significance of NTRK3 promoter methylation. a, b Kaplan-Meier curves of disease-free survival according to NTRK3 promoter methylation in CRC. Kaplan-Meier curves for the whole validation cohort (a) and stage I-III subgroup (b) were shown. The P value for each log-rank test was presented in the plots. Number at risk showed the quantity of CRC patients with NTRK3 hyper- or hypo- methylation and among them the quantity of survivors or dead patients respectively. $\mathbf{c}, \mathbf{d}$ A nomogram and calibration curve for predicting DFS in CRC. A nomogram to predict individual patient-level 3-year, and 5-year DFS based on clinicopathological risk factors and NTRK3 methylation (c). Calibration plots for the validation sample of the above nomogram (d). Actual DFS statue measured via Kaplan-Meier analysis is shown on the Y-axis, and the nomogram-predicted probability of DFS statue is shown on the X-axis. The average nomogram-predicted probability of DFS was plotted against actually observed DFS estimated by Kaplan-Meier. 95\% confidence intervals of the Kaplan-Meier estimates are indicated with vertical lines. Grayline indicates the reference line, showing where an ideal nomogram would lie. Instructions for users: Locate the status on each variable axis, and draw a straight line up to the Points axis to determine how many points toward risk the patient should receive from each variable. Sum the points and locate this number on the Total Points axis. Draw a straight line down from the total points to the 3-year or 5-year DFS Probability axis to ascertain the patient's specific possibility of maintaining DFS until 3 or 5 years. e, $\mathbf{f}$ Pan-cancer analysis of the prognostic significance of NTRK3 promoter methylation. The forest plots showed the values of the HR and Cl for the prediction of the survival outcomes in univariate Cox analysis for the methylation of cg27034819 (e) and cg11525479 (f) in 23 TCGA cancer types. The x-axes presenting HRs were log2-scaled 
a

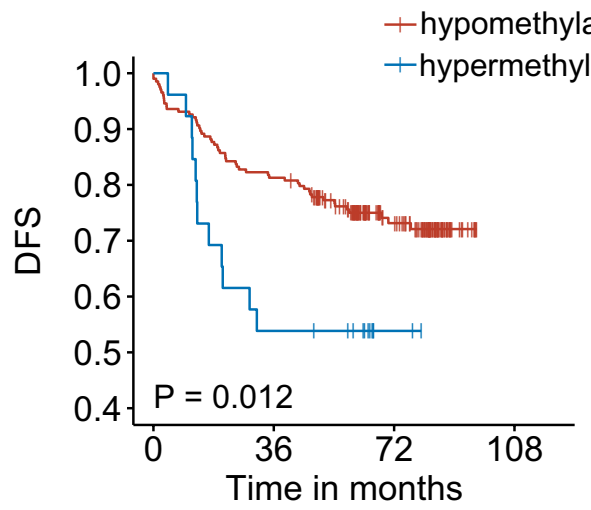

Number at risk

$\begin{array}{cccc}203 & 165 & 78 & 0 \\ 26 & 14 & 2 & 0\end{array}$

C

Points

$\begin{array}{llllllllll}0 & 10 & 20 & 30 & 40 & 50 & 60 & 70 & 80 & 90\end{array}$

NTRK3

Older age

TNM stage

BRAF mutation

Total Points

3-year DFS Probability

5-year DFS Probability

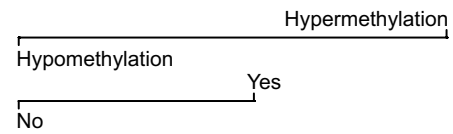

Stage III-IV

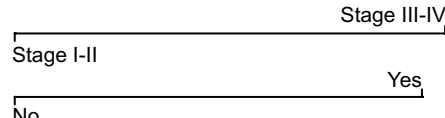

$\begin{array}{llllllll}0 & 40 & 80 & 120 & 160 & 200 & 240 & 280\end{array}$

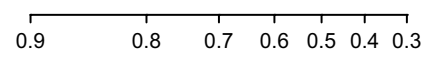

$\begin{array}{llllll}8 & 0.7 & 0.6 & 0.5 & 0.4 & 0.3\end{array}$

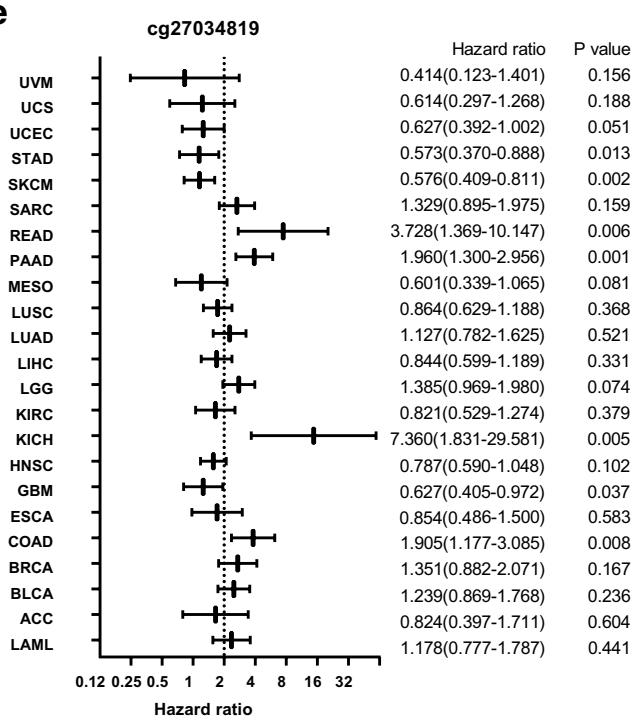

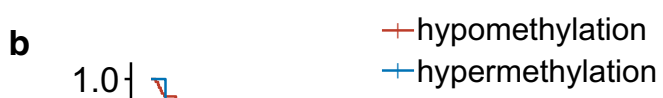

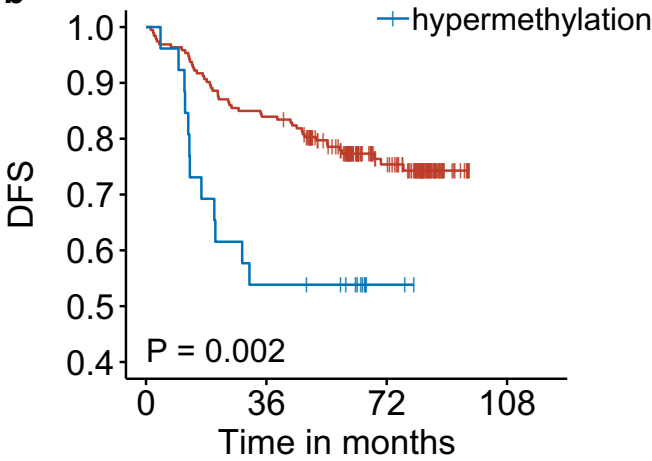

Number at risk

$\begin{array}{cccc}193 & 162 & 77 & 0 \\ 26 & 14 & 2 & 0\end{array}$

d

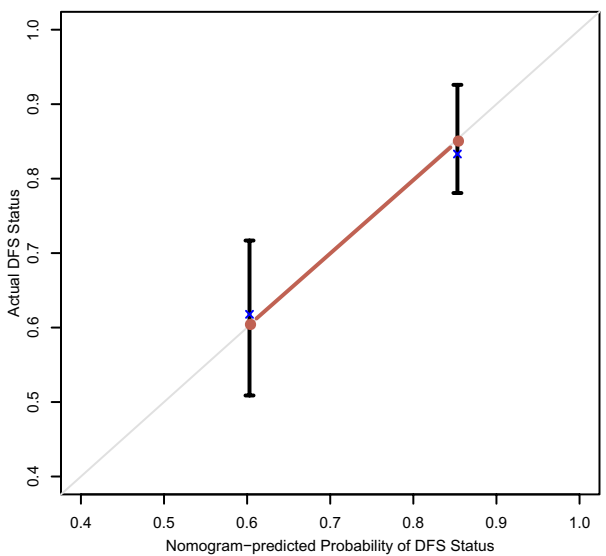

\section{f}

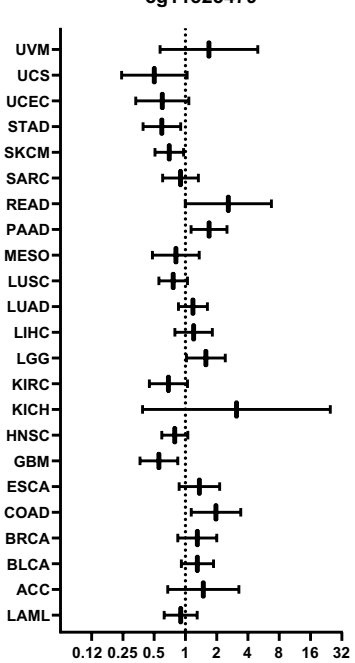

Hazard ratio $P$ value 1.682(0.570-4.963) $\quad 0.346$ $0.502(0.243-1.039) \quad 0.063$ $0.598(0.332-1.077) \quad 0.087$ $0.593(0.391-0.900) \quad 0.014$ $0.700(0.510-0.960) \quad 0.027$ $0.896(0.603-1.333) \quad 0.588$ $2.588(0.995-6.727) \quad 0.043$ 5) $\quad 0.010$ $0.809(0.482-1.358) \quad 0.422$ 0.762(0.555-1.047) $\quad 0.093$

$1.180(0.857-1.626) \quad 0.311$

$1.199(0.793-1.814) \quad 0.389$

$1.576(1.025-2.423) \quad 0.038$

$0.686(0.450-1.047) \quad 0.080$

$3.100(0.387-24.818) \quad 0.689$

$0.790(0.591-1.055) \quad 0.110$

$0.555(0.365-0.843) \quad 0.006$

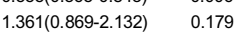

1.967(1.136-3.407) $\quad 0.014$

$1.301(0.845-2.006) \quad 0.232$

$1.305(0.914-1.863) \quad 0.142$

$1.486(0.676-3.266) \quad 0.324$

$\begin{array}{ll}0.901(0.625-1.300) & 0.578\end{array}$ 
Table 2 Cox proportional hazard analyses on DFS in patients with CRC

\begin{tabular}{|c|c|c|c|c|c|c|c|c|}
\hline \multirow[t]{3}{*}{ Variables } & \multicolumn{4}{|c|}{ DFS in the patients with I-IV stage CRC } & \multicolumn{4}{|c|}{ DFS in the patients with I-III stage CRC } \\
\hline & \multicolumn{2}{|c|}{ Univariate } & \multicolumn{2}{|c|}{ Multivariate } & \multicolumn{2}{|c|}{ Univariate } & \multicolumn{2}{|c|}{ Multivariate } \\
\hline & $P$ value & $\mathrm{HR}[95 \% \mathrm{Cl}]$ & $P$ value & $\mathrm{HR}[95 \% \mathrm{Cl}]$ & $P$ value & $\mathrm{HR}[95 \% \mathrm{Cl}]$ & $P$ value & $\mathrm{HR}[95 \% \mathrm{Cl}]$ \\
\hline NTRK3 hypermethylation & 0.014 & $2.194(1.169,4.117)$ & 0.004 & $2.688(1.355,5.333)$ & 0.004 & $2.565(1.354,4.857)$ & 0.015 & $2.630(1.206,5.734)$ \\
\hline Older age & 0.011 & $1.952(1.168,3.263)$ & 0.060 & $1.723(0.977,3.038)$ & 0.002 & $2.401(1.364,4.227)$ & 0.010 & $2.334(1.217,4.477)$ \\
\hline Male (vs. female) & 0.574 & $1.153(0.702,1.894)$ & & & 0.484 & $1.207(0.713,2.043)$ & & \\
\hline $\begin{array}{l}\text { Rectal tumor (vs. colon } \\
\text { tumor) }\end{array}$ & 0.479 & $1.192(0.733,1.939)$ & & & 0.341 & $1.087(0.915,1.291)$ & & \\
\hline Tumor size $\geq 4.5$ & 0.056 & $1.655(0.988,2.772)$ & & & 0.081 & $1.624(0.943,2.799)$ & & \\
\hline Poor differentiation & 0.581 & $1.193(0.637,2.233)$ & & & 0.553 & $1.220(0.633,2.353)$ & & \\
\hline Lymphovascular invasion & 0.052 & $2.087(0.995,4.378)$ & & & 0.022 & $2.387(1.131,5.038)$ & 0.003 & $3.287(1.465,7.373)$ \\
\hline Perineural invasion & 0.350 & $1.455(0.663,3.195)$ & & & 0.158 & $1.770(0.801,3.912)$ & & \\
\hline Advanced TNM stage & 0.003 & $2.125(1.300,3.473)$ & $<0.001$ & $2.704(1.528,4.790)$ & 0.019 & $1.854(1.107,3.103)$ & 0.039 & $1.978(1.034,3.784)$ \\
\hline CIMP positive status & 0.265 & $1.935(0.607,6.169)$ & & & 0.186 & $2.191(0.685,7.010)$ & & \\
\hline MSI & 0.316 & $1.350(0.751,2.427)$ & & & 0.138 & $1.599(0.860,2.973)$ & & \\
\hline KRAS mutation & 0.113 & $1.566(0.899,2.726)$ & & & 0.067 & $1.743(0.962,3.157)$ & & \\
\hline BRAF mutation & 0.010 & $3.376(1.338,8.519)$ & 0.049 & $2.563(1.004,6.551)$ & 0.003 & $4.122(1.621,10.485)$ & 0.595 & $1.443(0.373,5.591)$ \\
\hline $\mathrm{Ki}-67>25 \%$ & 0.458 & $0.806(0.456,1.425)$ & & & 0.675 & $0.877(0.476,1.617)$ & & \\
\hline CA19-9>37 & 0.078 & $1.780(0.938,3.377)$ & & & 0.191 & $1.589(0.794,3.179)$ & & \\
\hline$C E A>5$ & 0.095 & $1.591(0.923,2.741)$ & & & 0.048 & $1.769(1.005,3.111)$ & 0.772 & $1.122(0.514,2.447$ \\
\hline
\end{tabular}

NTRK3: neurotropic tropomyosin receptor kinase 3; CIMP: CpG island methylator phenotype; MSI: microsatellite instability; KRAS: kirsten rat sarcoma viral oncogene; BRAF: B-Raf proto-oncogene, serine/threonine kinase; Ki-67: kiel67 antigen; CA19-9: carbohydrate antigen 19-9; CEA: carcinoembryonic antigen

Table 3 Model fit among seven models including or not including NTRK3 methylation status

\begin{tabular}{lllll}
\hline Models & N & AIC & LR & P value \\
\hline Model 1 & 219 & 597.73 & 6.91 & \\
Model 2 & 219 & 600.69 & 5.95 & $0.005^{\mathrm{a}}$ \\
Model 3 & 219 & 592.41 & 16.23 & $0.002^{\mathrm{b}}$ \\
Model 4 & 219 & 516.49 & 39.06 & \\
Model 5 & 219 & 513.91 & 43.64 & $0.032^{\mathrm{c}}$ \\
\hline
\end{tabular}

$\mathrm{N}$ : patient counts in each model; AIC: Akaike information criterion value; LR: likelihood ratio

Model 1 includes NTRK3 hypermethylation

Model 2 includes TNM stage $(\mathrm{I}, \mathrm{II}, \mathrm{III})$

Model 3 includes TNM stage (I, II, III), NTRK3 hypermethylation

Model 4 includes age at diagnosis, sex (m/f), location(left colon, right colon, rectum), differentiation (well, moderate, poor), lymphovascular invasion, perineural invasion $(\mathrm{n} / \mathrm{y})$, T stage $(\mathrm{T} 1, \mathrm{~T} 2, \mathrm{~T} 3, \mathrm{~T} 4), \mathrm{N}$ stage (N0, N1, N2), negative lymph node number, preoperation CEA, chemotherapy(n/y)

Model 5 includes NTRK3 hypermethylation and all variables in Model 4

a $P$ values for the LR test in model 1 compared with model $2{ }^{b} P$ values for the LR test in model 2 compared with model $3 ;{ }^{C} P$ values for the LR test in model 4 compared with model 5

(AIC: 600.69 vs 592.41; LR: 5.95 vs $16.23, P=0.002$; Table 3). These results suggest NTRK3 hypermethylation could increase prognostic values of TNM staging.

To determine the values of NTRK3 hypermethylation in commonly-used models using multiple clinicopathological variables, model 4 was built using the variables included in the model recommended by AJCC [47, 48]. As expected, after NTRK3 hypermethylation was included, model 5 had a lower AIC and a higher LR in comparison to model 4 (AIC: 516.49 vs 513.91; LR: 39.06 vs 43.64, $P=0.032$; Table 3 ). Thus, the model recommended by AJCC may get increased discriminatory ability in predicting prognosis with NTRK3 hypermethylation.

\section{Prognostic significance of NTRK3 methylation in multiple tumors}

Both the methylation of cg27034819 and cg11525479 were analyzed on their associations with survival outcome in 23 tumors using TCGA methylation profiles generated by $450 \mathrm{~K}$ microarray. Overall, similar to the conflicting findings from in vitro and in vivo studies on NTRKs gene, the association of their methylations with survival outcome varied in different tumors. The hypermethylation of cg27034819 was significantly associated with worse survival outcome in colon adenocarcinoma (COAD; $P=0.008$, HR 1.91, 95\% CI [1.18, 3.09]), rectum adenocarcinoma (READ; $P$ 0.006, HR 3.73, 95\% CI $[1.37,10.15])$, kidney chromophobe (KICH; $P=0.005$, HR 7.36, 95\% CI [1.83, 29.581], and pancreatic adenocarcinoma (PAAD; $P=0.001, \mathrm{HR} 1.96,95 \%$ CI [1.30, 2.96]) cohorts. However, it was significantly associated 
with better survival outcome in glioblastoma multiforme (GBM; $P=0.037$, HR 0.63, 95\% CI [0.41, 0.97], skin cutaneous melanoma (SKCM; $P=0.002$, HR 0.58, 95\% $\mathrm{CI}[0.41,0.81])$ and stomach adenocarcinoma (STAD; $P=0.013$, HR 0.57, 95\% CI [0.37, 0.89]) cohorts (Fig. 3e). In cg11525479 methylation analysis, a similar predictive value for worse survival were found in COAD, READ, and PAAD, and a similar predictive value for better survival were found in GBM, SKCM and STAD (Fig. 3f). These results suggested a robust prognostic value of the methylation of the specific promoter region targeted by cg27034819 and cg11525479 in multiple tumors.

\section{Discussions}

In this study, we found the NTRKs gene promoter was more frequently methylated in CRC compared to normal mucosa, which was associated with suppressed expression of NTRK 2 and NTRK3. Through a screen of probes targeting NTRKs gene, we identified a specific methylated region within NTRK3 promoter targeted by cg27034819 and cg11525479 that was the most promising prognostic marker for CRC. We developed a QMSP assay to determine the methylation of this region that could be easily applied in clinical assay and validate its predictive value for survival outcome in a cohort of 229 CRC patients and 23 TCGA cohorts including a colon cancer cohort, a rectal cancer cohort and 21 cohorts of other tumor types. Using NTRK3 promoter methylation, age, TNM stage, and $B R A F$ mutation, a novel nomogram predicting DFS outcome was developed and validated with a good prognostic performance. Also, we investigated the values of NTRK3 promoter methylation that added to current prognostic panels, in which we observed a meaningful performance improvement of AJCC model and TNM staging alone after the introduction of NTRK3 promoter methylation.

The conflicting findings in previous studies have revealed the complicated roles of NTRKs in different cancers. In our results, the mRNA expression of NTRK2 and NTRK3 was commonly lower in CRC samples in comparison to normal tissues, while the difference was not observed for NTRK1. In addition, this decreased expression of NTRKs was associated with promoter methylation. These results indicated that NTRK2 and NTRK3 may play a more important role of tumor suppressor in CRC, and methylation silencing of NTRK2 and NTRK3 would contribute more to CRC tumorigenesis. However, a decreased mRNA expression of NTRK1 was found and attributed to the methylated promoter in neuroblastoma and ovarian cancer $[33,35]$.

In the discovery set, we identified the CpG site targeted by cg27034819 as the most promising methylation biomarker for prognosis, and further assessed its prognostic value in other cohorts. To apply this high-throughput screen finding to a clinical assay in large cohorts of patients, we developed a PCR-based assay covering this genomic region that can be easily used with low cost. The robustness of this assay includes the finding that the probe cg11525479 that was very close to cg27034819 also had a prognostic value superior to most probes. The use of this assay in our previous in vitro study on NTRK3 also strengthen the reliability [11]. Using this assay in our CRC cohort, we found that NTRK3 promoter hypermethylation was associated with worse DFS validation. This association maintained after the adjustment with all clinicopathological predictors. Furthermore, we revealed that NTRK3 promoter hypermethylation is highly associated with MSI and KRAS mutation that is known as response biomarkers for cancer treatment and have conflicting predictive value for survival [4]. Therefore, we conducted sensitivity analyses excluding the patients with MSI or KRAS mutation. Consequently, the adverse impact of these molecular phenotypes on our methylation biomarker was minimal or not observed. Therefore, we are convinced that NTRK3 promoter methylation determined by the QMSP assay we developed was an independent prognostic factor in CRC.

We used AIC and likelihood-ratio test to compare the discriminatory ability of predictive models for survival outcome as previously described [49]. First, NTRK3 methylation status is better than rough TNM stage (I, II, III) in predicting prognosis in CRC. Then, the addition of NTRK3 promoter methylation status in TNM stage and the AJCC models was shown to improve the predictive performance for DFS in CRC patients. Thus, NTRK3 promoter methylation is a valuable prognostic marker in CRC patients.

In previous studies, NTRK3 has been demonstrated to be an oncogene or a tumor suppressor gene in different cancer types [11, 28-31, 50, 51]. These conflicting findings from in vitro and in vivo studies is similar to the results of our cohort analyses on NTRK3 methylation. In our analyses, NTRK3 hypermethylation was associated with worse survival in some tumors, such as CRC, kidney chromophobe, and pancreatic adenocarcinoma, but it is related to a better outcome in other tumors, including glioblastoma multiforme, skin Cutaneous Melanoma, and stomach adenocarcinoma. NTRK3 promoter methylation could serve as a prognostic marker in multiple cancers, and its prognosis role is different in various cancers.

We have to admit that our study has some limitations. First, despite the multiple supports from discovery, SYSU and TCGA cohorts, external validation for NTRK3 promoter methylation and the novel nomogram using the QMSP assay we developed would strengthen our findings. In addition, an independent validation in the cohort 
with methylation and transcriptomic profiles is essential for the association of NTRKs gene promoter methylation with its mRNA expression. Finally, although we excluded patients receiving chemo/radiotherapy before sample collection (neoadjuvant treatment) to avoid the confounding effect on genomic methylation and clonal selection, the retrospective nature of the SYSU cohort does not totally rule out other potential confounding factors.

\section{Conclusions}

We found NTRKs gene was commonly suppressed by promoter methylation in CRC compared to normal mucosa. We identified the $\operatorname{cg} 27034819-\operatorname{cg} 11525479$ region within NTRK3 promoter as the most promising predictive marker for survival outcome, and it was validated in our CRC cohort and 23 TCGA cohorts including a colon cancer cohort, a rectal cancer cohort and 21 cohorts of other tumor types. A novel nomogram included NTRK3 promoter methylation and other independent predictors was developed. In addition, we observed a performance improvement of currently used prognostic models after the introduction of NTRK3 promoter methylation. These findings have essential implications for prognosis stratification in clinical decision-making for CRC management.

\section{Supplementary Information}

The online version contains supplementary material available at https://doi. org/10.1186/s12967-021-02740-6.

Additional file 1. Table S1. Primer and Probe Sequences. Table S2. The mean methylation of NTRKs promoter in CRC samples and matched normal mucosae in FHCRC cohort. Table S3. Univariate Cox analysis of probes targeting NTRKs gene in TCGA-COADREAD cohort. Table S4. Sensitivity analysis excluding patients with CIMP-positive status. Table S5. Sensitivity analysis excluding patients with MSI. Table S6. Sensitivity analysis excluding patients with BRAF mutation. Table S7. Sensitivity analysis excluding patients with KRAS mutation.

\section{Abbreviations}

ACC: Adrenocortical carcinoma; AIC: Akaike information criterion; BLCA: Bladder urothelial carcinoma; BRAF: B-Raf proto-oncogene, serine/threonine kinase; BRCA: Breast invasive carcinoma; CA 19-9: Carbohydrate antigen 19-9; CEA: Carcinoembryonic antigen; Cl: Confidence intervals; CIMP: CpG island methylator phenotype; C-index: Concordance index; COAD: Colon adenocarcinoma; COADREAD: Colorectal adenocarcinoma; CRC: Colorectal cancer; DFS: Disease-free survival; ESCA: Esophageal carcinoma; FFPE: Formalin-fixed paraffin-embedded; FHCRC: Fred Hutchinson Cancer Research Center; GBM: Glioblastoma multiforme; HNSC: Head and neck squamous cell carcinoma; HR: Hazard ratio; Ki-67: Kiel67 antigen; KICH: Kidney chromophobe; KIRC: Kidney renal clear cell carcinoma; KRAS: Kirsten rat sarcoma viral oncogene; LAML: Acute myeloid leukemia; LGG: Brain lower grade glioma; LIHC: Liver hepatocellular carcinoma; LR: Likelihood ratio; LUAD: Lung adenocarcinoma; LUSC: Lung squamous cell carcinoma; MESO: Mesothelioma; MSI: Microsatellite instability; NTRK1: Neurotropic tropomyosin receptor kinase 1; NTRK2: Neurotropic tropomyosin receptor kinase 2; NTRK3: Neurotropic tropomyosin receptor kinase 3; NTRKs: Neurotropic tropomyosin receptor kinases; PAAD: Pancreatic adenocarcinoma; PMR: Percentage of methylated reference; QMSP: Quantitative methylation-specific PCR; READ: Rectum adenocarcinoma; SARC: Sarcoma; SKCM: Skin cutaneous melanoma; STAD: Stomach adenocarcinoma; SYSU:
Sun Yat-sen University; TCGA: The Cancer Genome Atlas; TRK: Tropomyosin receptor kinases; UCEC: Uterine corpus endometrial carcinoma; UCS: Uterine carcinosarcoma; UVM: Uveal melanoma.

\section{Acknowledgements}

We thank all patients for participation in this study.

\section{Authors' contributions}

Collection of data: ZC, ZH, HY, QZ, LB, XW, GT, XF, MH; data analyses: ZC, HY; manuscript writing: ZC, ZH, HY, MH, JW, GC; Supervision, study designation and manuscript editing: $H Y, J X$ and $Y L$. All authors read and approved the final manuscript.

\section{Funding}

This research was funded by the National Basic Research Program of China (No. 2015CB554001, JW), the National Natural Science Foundation of China (No. 81972245, YL; No. 81902877, HY), the Natural Science Fund for Distinguished Young Scholars of Guangdong Province (No. 2016A030306002,

$\mathrm{YL}$ ), the Natural Science Foundation of Guangdong Province (No. 2016A030310222, HY; No. 2018A0303130303, HY), Sun Yat-Sen University Clinical Research 5010 Program (No. 2018026, YL), the "Five Five"Talent Team Construction Project of the Sixth Affiliated Hospital Of Sun Yat-Sen University (No. P20150227202010244, JW; No. P20150227202010251, YL), the Program of Introducing Talents of Discipline to Universities, and National Key Clinical Discipline (2012).

\section{Availability of data and materials}

The datasets used during the current study are available from the corresponding author on reasonable request.

\section{Ethics approval and consent to participate}

Ethical approval was given by the Institutional Review Board at the Sixth Affiliated Hospital of Sun Yat-sen University. All the included patients have been given the written informed consent.

\section{Consent for publication}

Informed consent was obtained from all individual participants included in the study, giving their authorization to access their clinical information and tumor samples for research purpose.

\section{Competing interests}

The authors declared no potential conflicts of interest with respect to the research, authorship, and publication.

\section{Author details}

${ }^{1}$ Department of Gastrointestinal Surgery, The Sixth Affiliated Hospital, Sun Yat-sen University, Guangzhou, China. ${ }^{2}$ Department of Colorectal Surgery, The Sixth Affiliated Hospital, Sun Yat-sen University, Guangzhou, China. ${ }^{3}$ Guangdong Institute of Gastroenterology, Guangdong Provincial Key Laboratory of Colorectal and Pelvic Floor Disease, The Sixth Affiliated Hospital, Sun Yat-sen University, Guangzhou, China. ${ }^{4}$ Department of Colorectal and Anal Surgery, The Sixth Affiliated Hospital, Sun Yat-sen University, Guangzhou, China. ${ }^{5}$ Department of Epidemiology, Second Military Medical University, Shanghai, China.

Received: 6 November 2020 Accepted: 4 February 2021

Published online: 16 February 2021

\section{References}

1. Bray F, Ferlay J, Soerjomataram I, Siegel RL, Torre LA, Jemal A. Global Cancer Statistics 2018: GLOBOCAN estimates of incidence and mortality worldwide for 36 cancers in 185 countries. Cancer J Clin. 2018;68(6):394-424

2. Torre LA, Bray F, Siegel RL, Ferlay J, Lortet-Tieulent J, Jemal A. Global cancer statistics, 2012. CA Cancer J Clin. 2015;65(2):87-108.

3. Mahar AL, Compton C, Halabi S, Hess KR, Weiser MR, Groome PA. Personalizing prognosis in colorectal cancer: a systematic review of the quality 
and nature of clinical prognostic tools for survival outcomes. J Surg Oncol. 2017;1 16(8):969-82.

4. Kocarnik JM, Shiovitz S, Phipps Al. Molecular phenotypes of colorectal cancer and potential clinical applications. Gastroenterol Rep. 2015;3(4):269-76

5. Abdel Ghafar MT, Gharib F, Abdel-Salam S, Elkhouly RA, Elshora A, Shalaby $\mathrm{KH}$, et al. Role of serum Metadherin mRNA expression in the diagnosis and prediction of survival in patients with colorectal cancer. Mol Biol Rep. 2020:47(4):2509-19.

6. Yurgelun MB, Kulke MH, Fuchs CS, Allen BA, Uno H, Hornick JL, et al. Cancer susceptibility gene mutations in individuals with colorectal cancer. J Clin Oncol. 2017:35(10):1086-95.

7. Wolff RK, Hoffman MD, Wolff EC, Herrick JS, Sakoda LC, Samowitz WS, et al. Mutation analysis of adenomas and carcinomas of the colon: early and late drivers. Genes Chromosomes Cancer. 2018;57(7):366-76.

8. Esteller M. Epigenetics in cancer. N Engl J Med. 2008;358(11):1148-59.

9. Chang SY, Kuo CC, Wu CC, Hsiao CW, Hu JM, Hsu CH, et al. NKX6.1 hypermethylation predicts the outcome of stage II colorectal cancer patients undergoing chemotherapy. Genes Chromosomes Cancer. 2018:57(5):268-77.

10. Graule J, Uth K, Fischer E, Centeno I, Galvan JA, Eichmann M, et al. CDX2 in colorectal cancer is an independent prognostic factor and regulated by promoter methylation and histone deacetylation in tumors of the serrated pathway. Clin Epigenet. 2018:10(1):120.

11. Luo Y, Kaz AM, Kanngurn S, Welsch P, Morris SM, Wang J, et al. NTRK3 is a potential tumor suppressor gene commonly inactivated by epigenetic mechanisms in colorectal cancer. PLoS Genet. 2013;9(7):e1003552.

12. Khotskaya YB, Holla VR, Farago AF, Mills Shaw KR, Meric-Bernstam F, Hong DS. Targeting TRK family proteins in cancer. Pharmacol Ther. 2017;173:58-66.

13. Scott L. Larotrectinib: first global approval. Drugs. 2019;79(2):201-6.

14. Cho YA, Chung JM, Ryu H, Kim EK, Cho BC, Yoon SO. Investigating Trk protein expression between oropharyngeal and non-oropharyngeal squamous cell carcinoma: clinical implications and possible roles of human papillomavirus infection. Cancer Res Treat. 2019;51(3):1052-63.

15. Kamiya A, Inokuchi M, Otsuki S, Sugita H, Kato K, Uetake H, et al. Prognostic value of tropomyosin-related kinases $A, B$, and $C$ in gastric cancer. Clin Transl Oncol. 2016;18(6):599-607.

16. Leveque R, Corbet $C$, Aubert L, Guilbert M, Lagadec C, Adriaenssens $E_{,}$ et al. ProNGF increases breast tumor aggressiveness through functional association of TrkA with EphA2. Cancer Lett. 2019;449:196-206.

17. Xu Z. Tumor-derived exosomes educate fibroblasts to promote salivary adenoid cystic carcinoma metastasis via NGF-NTRK1 pathway. Oncol Lett. 2019;18(4):4082-91.

18. Yang $X$, Shen H, Buckley B, Chen Y, Yang N, Mussell AL, et al. NTRK1 is a positive regulator of YAP oncogenic function. Oncogene. 2019;38(15):2778-87.

19. Youssef G, Gillett C, Rampling D, Chagtai T, Virasami A, Barton J, et al. The presence of Y674/Y675 phosphorylated NTRK1 via TP53 repression of PTPN6 expression as a potential prognostic marker in neuroblastoma. Hum Pathol. 2019:86:182-92.

20. Xu Y, Jiang WG, Wang HC, Martin T, Zeng YX, Zhang J, Qi YS. BDNF activates TrkB/PLCY1 signaling pathway to promote proliferation and invasion of ovarian cancer cells through inhibition of apoptosis. Eur Rev Med Pharmacol Sci. 2019;23:5093-100.

21. Zhou Y, Sinha S, Schwartz JL, Adami GR. A subtype of oral, laryngeal, esophageal, and lung, squamous cell carcinoma with high levels of TrkBT1 neurotrophin receptor mRNA. BMC Cancer. 2019;19(1):607.

22. Contreras-Zarate MJ, Day NL, Ormond DR, Borges VF, Tobet S, Gril B, et al. Estradiol induces BDNF/TrkB signaling in triple-negative breast cancer to promote brain metastases. Oncogene. 2019;38(24):4685-99.

23. Li W, Lu Y, Yu X, Yong M, Ma D, Gao Q. Detection of exosomal tyrosine receptor kinase B as a potential biomarker in ovarian cancer. J Cell Biochem. 2019;120(4):6361-9.

24. Wang X, Xu Z, Chen X, Ren X, Wei J, Zhou S, et al. A tropomyosin receptor kinase family protein, NTRK2 is a potential predictive biomarker for lung adenocarcinoma. PeerJ. 2019;7:e7125.

25. Deluche E, Bessette B, Durand S, Caire F, Rigau V, Robert S, et al. CHI3L1, NTRK2, 1p/19q and IDH status predicts prognosis in glioma. Cancers. 2019;11(4):544.
26. Meng L, Liu B, Ji R, Jiang $X$, Yan $X$, Xin Y. Targeting the BDNF/TrkB pathway for the treatment of tumors. Oncol Lett. 2019;17(2):2031-9.

27. Thomaz A, Pinheiro KV, Souza BK, Gregianin L, Brunetto AL, Brunetto AT, et al. Antitumor activities and cellular changes induced by TrkB inhibition in medulloblastoma. Front Pharmacol. 2019;10:698.

28. Jin W, Kim GM, Kim MS, Lim MH, Yun C, Jeong J, et al. TrkC plays an essential role in breast tumor growth and metastasis. Carcinogenesis. 2010;31(11):1939-47.

29. Bu JY, Lv WZ, Liao YF, Xiao XY, Lv BJ. Long non-coding RNA LINC00978 promotes cell proliferation and tumorigenesis via regulating microRNA-497/NTRK3 axis in gastric cancer. Int J Biol Macromol. 2019:123:1106-14.

30. Bouzas-Rodriguez J, Cabrera JR, Delloye-Bourgeois C, Ichim G, Delcros JG, Raquin MA, et al. Neurotrophin-3 production promotes human neuroblastoma cell survival by inhibiting TrkC-induced apoptosis. J Clin Invest. 2010;120(3):850-8.

31. Foerster $Y$, Stover T, Wagenblast J, Diensthuber M, Balster S, Gabrielpillai J, et al. Relevance of neurotrophin receptors CD271 and TrkC for prognosis, migration, and proliferation in head and neck squamous cell carcinoma. Cells. 2019:8(10):1167.

32. Behrouz Sharif S, Hashemzadeh S, Mousavi Ardehaie R, Eftekharsadat A, Ghojazadeh M, Mehrtash AH, et al. Detection of aberrant methylated SEPT9 and NTRK3 genes in sporadic colorectal cancer patients as a potential diagnostic biomarker. Oncol Lett. 2016;12(6):5335-43.

33. Li Z, Takenobu H, Setyawati AN, Akita N, Haruta M, Satoh S, et al. EZH2 regulates neuroblastoma cell differentiation via NTRK1 promoter epigenetic modifications. Oncogene. 2018;37(20):2714-27.

34. Palani M, Arunkumar R, Vanisree AJ. Methylation and expression patterns of tropomyosin-related kinase genes in different grades of glioma. Neuromol Med. 2014;16(3):529-39.

35. Chmelarova M, Dvorakova E, Spacek J, Laco J, Mzik M, Palicka V. Promoter methylation of GATA4, WIF1, NTRK1 and other selected tumour suppressor genes in ovarian cancer. Folia Biol. 2013;59(2):87-92.

36. Yamada Y, Toyota M, Hirokawa Y, Suzuki H, Takagi A, Matsuzaki T, et al. Identification of differentially methylated $\mathrm{CpG}$ islands in prostate cancer. Int J Cancer. 2004;112(5):840-5.

37. Cancer Genome Atlas. Comprehensive molecular characterization of human colon and rectal cancer. Nature. 2012:487(7407):330-7.

38. Kwon Y, Park M, Jang M, Yun S, Kim WK, Kim S, et al. Prognosis of stage III colorectal carcinomas with FOLFOX adjuvant chemotherapy can be predicted by molecular subtype. Oncotarget. 2017;8(24):39367-81.

39. Hu Y, Gaedcke J, Emons G, Beissbarth T, Grade M, Jo P, et al. Colorectal cancer susceptibility loci as predictive markers of rectal cancer prognosis after surgery. Genes Chromosomes Cancer. 2018;57(3):140-9.

40. Marisa L, de Reynies A, Duval A, Selves J, Gaub MP, Vescovo L, et al. Gene expression classification of colon cancer into molecular subtypes: characterization, validation, and prognostic value. PLoS Med. 2013;10(5):e1001453.

41. Deng Y, Chi P, Lan P, Wang L, Chen W, Cui L, et al. Neoadjuvant modified FOLFOX6 with or without radiation versus fluorouracil plus radiation for locally advanced rectal cancer final results of the Chinese FOWARC Trial. J Clin Oncol. 2019;37(34):3223-33.

42. Benson AB, Venook AP, Al-Hawary MM, Cederquist L, Chen YJ, Ciombor KK, et al. NCCN Guidelines Insights: colon Cancer, Version 2.2018. J Natl Compr Canc Netw. 2018;16(4):359-69.

43. Benson AB, Venook AP, Al-Hawary MM, Arain MA, Chen YJ, Ciombor KK, et al. NCCN Guidelines Insights: rectal Cancer, Version 6.2020. J Natl Compr Canc Netw. 2020;18(7):806-15.

44. Yu H, Bai L, Tang G, Wang $X$, Huang M, Cao G, et al. Novel assay for quantitative analysis of DNA methylation at single-base resolution. Clin Chem. 2019;65(5):664-73.

45. Luo Y, Tsuchiya KD, II Park D, Fausel R, Kanngurn S, Welcsh P, et al. RET is a potential tumor suppressor gene in colorectal cancer. Oncogene. 2012:32:2037.

46. Fu XH, Chen ZT, Wang WH, Fan XJ, Huang Y, Wu XB, et al. KRAS G12V mutation is an adverse prognostic factor of Chinese gastric cancer patients. J Cancer. 2019;10(4):821-8.

47. Weiser MR, Landmann RG, Kattan MW, Gonen M, Shia J, Chou J, et al. Individualized prediction of colon cancer recurrence using a nomogram. J Clin Oncol. 2008;26(3):380-5. 
48. Weiser MR, Gönen M, Chou JF, Kattan MW, Schrag D. Predicting survival after curative colectomy for Cancer: individualizing colon cancer staging. J Clin Oncol. 2011;29(36):4796-802.

49. Deckers IA, Schouten LI, Van Neste L, van Vlodrop IJ, Soetekouw PM, Baldewijns MM, et al. Promoter methylation of CDO1 identifies clear-cell renal cell cancer patients with poor survival outcome. Clin Cancer Res. 2015;21(15):3492-500

50. Zhao S, Fan NF, Chen XH, Zhuo CH, Xu CW, Lin RB. Long noncoding RNA PVT1-214 enhances gastric cancer progression by upregulating TrkC expression in competitively sponging way. Eur Rev Med Pharmacol Sci. 2019;23:4173-84.
51. Marino FZ, Pagliuca F, Ronchi A, Cozzolino I, Montella M, Berretta M, et al. NTRK fusions, from the diagnostic algorithm to innovative treatment in the era of precision medicine. Int J Mol Sci. 2020;21(10):3718.

\section{Publisher's Note}

Springer Nature remains neutral with regard to jurisdictional claims in published maps and institutional affiliations.
Ready to submit your research? Choose BMC and benefit from:

- fast, convenient online submission

- thorough peer review by experienced researchers in your field

- rapid publication on acceptance

- support for research data, including large and complex data types

- gold Open Access which fosters wider collaboration and increased citations

- maximum visibility for your research: over $100 \mathrm{M}$ website views per year

At BMC, research is always in progress.

Learn more biomedcentral.com/submissions 